\title{
Fatigue Analysis of Ti-55 Alloy Material for Multifunctional Surgical Instruments in Medical Engineering
}

\author{
Ö. KARAÇALI* \\ Department of Mechanical Engineering, Faculty of Engineering, Istanbul University, Avc1lar, Istanbul, 34320 Turkey
}

\begin{abstract}
A new approach is proposed for fatigue prediction in medical engineering of multifunctional microforcepsscissors (MFS), transconjunctival sutureless vitroretinal metal surgical instrument that removes, grasp, chops tissues and inserts new in a cell. Cyclic loads can result in the fatigue failure of MFS at stress levels below the yielding stress of material. Hence, research of the material and mechanical behavior of a MFS structure under a cyclic load is required. The numerical modeling of a cyclic load fatigue test was performed for a biocompatible MFS, by using finite element analysis (FEA). To verify the data gathered from von Mises' yield condition, Haigh diagram was developed to predict fatigue life. Fatigue behavior of the MFS was analyzed in ANSYS LS-DYNA under operation load conditions in vitrectomy. This research analyzes the application of forces to the examined MFS, resulting in different movements, which cause fatigue during surgery. The numerical analysis consisted in solving the strains and stresses distribution in the operating part of the MFS, made of Ti-55 alloy ( $\mathrm{Ti}-5 \mathrm{Al}-4 \mathrm{Sn}-$ $2 \mathrm{Zr}-1 \mathrm{Mo}-0.25 \mathrm{Si}-1 \mathrm{Nd}$ ) medical material. The maximum values of strain and stress were calculated for the Ti-55 alloy, using values of elasticity modulus of $119 \mathrm{GPa}$, and Poisson's ratio of 0.32 . The outcomes of the fatigue analysis from this research will be beneficial for micro component manufacturers in medical engineering and for clinic surgeons.
\end{abstract}

DOI: 10.12693/APhysPolA.127.1199

PACS: 81.40.Np, 81.05.Bx, 87.10.Kn

\section{Introduction}

Retinal microsurgery requires extremely delicate manipulation of retinal tissue, where tool-to-tissue interaction forces trigger the fatigue and fracture on the tips of micro forceps. Imprecise movements of micro forceps due to fatigue and cracks often may result in tissue damage, which can be irreversible, and sight-threatening, depending on the location. Fatigue evaluation of biocompatible titanium micro materials and micro parts such as micro forceps scissors has been increasing in importance in recent years, in a variety of fields [1]. These include the development of new materials such as medical biocompatible titanium metal device materials; the practical application of micro machines; and the increasing demand for small and highly functional parts for the biomaterial titanium devices for medical surgeries [2]. The single most important factor that distinguishes titanium biomaterial and its alloys from any other material, is its ability to exist in contact with tissues of the human body, without causing unacceptable harm to that body [3]. Titanium alloys are widely used because of the desirable properties of the material, such as relatively low modulus, good fatigue strength, formability, machinability, corrosion resistance, and biocompatibility. However, titanium and its alloys can not meet all of the clinical requirements $[4,5]$. Therefore, in order to meet such material and mechanical requirements, a fatigue investigation of multifunctional forceps-scissors under a cyclic load was carried out in this research.

*e-mail: ozdogank@istanbul.edu.tr

\section{Materials and methods}

Handling of micro parts or components, such as multifunctional forceps-scissor (MFS), is a great challenge due to their small dimensions. The state of knowledge in macro technologies is not smoothly applicable to the micro world, because of the dimension effects and the related modified behavior of parts. Although, size constrains change the mechanical properties (e.g. in MFS the square cross section of one forceps leg is $1 \times 0.5 \mathrm{~mm}^{2}$ ), as well as the fatigue behavior, no significant research has been done on small-scale multi-axial fatigue analysis. The research focus of this experimental work lies on the multi-axial fatigue behavior of small forceps. Multi-axial fatigue experiment with micro forceps at $\mathrm{Hz}$ frequencies requires a commercial fatigue testing system for cyclic loading employed in laboratory. Fatigue testing is used to apply a cyclical load to a test specimen in order to better understand how that material will behave under similar loading cycles in a real world application (Fig. 1). Manual action occurs by compressing thin-walled metal ribs comprising its body, the outer tips of the MFS then move forward to engage the two "finger tips" of the forceps as demonstrated in Fig. 1. The applied load is often repeated until failure, with frequency of several hundred loading cycles per second. Testing was was carried out on a simulated specimen made of Ti-55 alloy, according to ASTM E1823-13 standard, which specifies the method to determine the upper and lower plateau strengths, tensile strength and elongation of the Ti- 55 material. Fatigue testing and analysis was conducted according to the ISO5832-3 for Surgery-Metallic Materials-Part 3, ISO 6892 for Metallic Materials-Tensile Testing and ISO9001. 


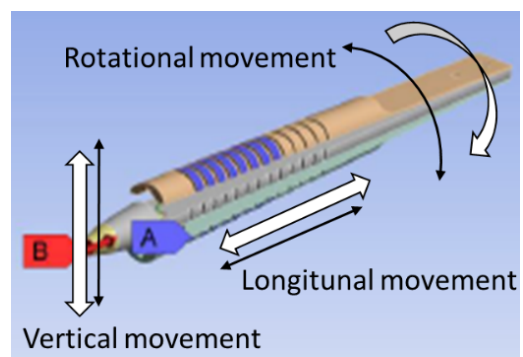

Fig. 1. Forces applied to the working model of MFS.

The current research work is focused on identifying these critical levels of damage and in characterizing the fatigue behavior under representative operating conditions in Ti-55 alloy, used in the multifunctional forcepsscissors. During surgical procedure on retina, MFS requires unimanual control, bimanual control or double unimanual control, when each hand holds an instrument simultaneously, for example a dissecting forceps and needle, a chisel. In vitreoretinal surgery, surgeons create two insertion ports in the eye to insert the trocars. Surgical instruments are inserted through the trocars to reach the target area on the eye ground. The forces of interest in vitreoretinal surgery are mainly the forces which appear after the tissue is grasped. This requires the MFS to be as much strain-free as possible, while the tips of forceps are closed. In designing forceps, there exist two main design approaches, depending on the neutral position of the grasping tips: closed design vs. open configuration. The MFS developed in this research is of closed design, to reserve the limited strain range only for tool-to-tissue interaction forces, and not to waste it by inner forces. This research analyzes the application of force resulting in three different movement of the examined MFS, as shown in Fig. 1. According to the type of motion, forces also may be classified for possible action of fatigue.

The longitudinal adjustment (the first drive, the handle of which is mounted on the main body of microforceps) controls the closure of the parts. During this motion the opening-closing function on the tips of the MFS takes place. In the present study we are using the threedimensional finite element analysis results based on tensile strength deformation in tips made of Ti-55 material.

During the vertical action or locking action of the endpart of the tips the force is transferred to the links by the movement of a pipe-piece. The blades of the closing jaw of the forceps apply the final portion of the pushing force. The smallest necessary pushing force for the implementation of the closure of the two extremities can be established by reducing the force applied to the main body of MFS. This is the push force at the tip-end portion of the tube, that exceed the sum of frictional force and the force required for closing of two blades. Grasping, cutting, clamping, probing, and other surgical maneuvers of MFS require specific ways of holding the instrument, with specific positions and actions of the fingers, hand, wrist, forearm, elbow. In this research, variable or "uncontrolled" cycling forces had been applied to standardized multifunctional forceps-scissors, causing the fatigue.

The rotational motion produces the friction force (between the tissue and the surgical instrument), which results in closing of the tips. As a result the rotational movement of MFS in the retina will determine the upper and lower force limits, at which the affected area of the retinal membrane will be peeled off, by such motion of MFS. The structure of the MFS is subjected to fluctuating bending, torsion and axial loading, as a result of the surgical motion of the instruments during the intraocular surgery.

In this study, two different types of loading were employed: (i) the internal pressure loading only and (ii) combined internal pressure and axial loading. The effects of friction, deformations on stress behavior in the MFS component were examined, for a period of fifty six seconds (Fig. 2). The effects of internal pressure alone and of the combined internal pressure and axial compressive load are investigated in this study. It is found that the development of stress and the location of maximum stress were dependent on the type of loading applied by fingers of a surgeon.

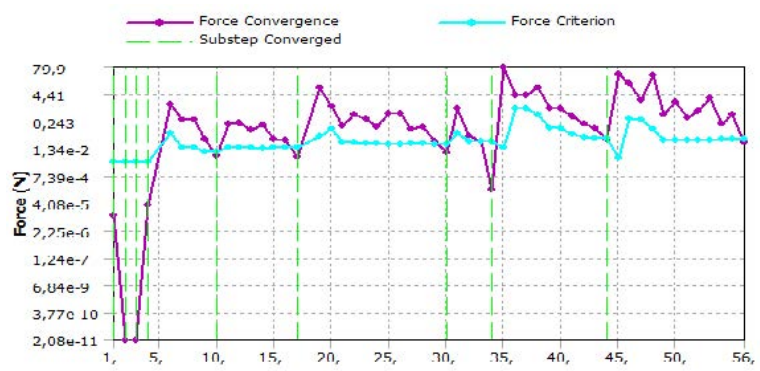

Fig. 2. Stresses occurred in MFS as function of time iteration.

In this research, numerical simulation for MFS has been performed with the ANSYS LS-DYNA software and material model for MFS has been established. Threedimensional solid models of the MFS were first created in the SolidWorks, CAD software, and then imported into the ANSYS Workbench, CAE software, with the use of the Parasolid format. For evaluation of the MFS Ti55 alloy, in this study $30 \mathrm{~Hz}$ stress-life tests were performed, with maximum mean stress of $500 \mathrm{MPa}$. The finite element simulation of MFS working model, using Von Mises method was carried out. Results are shown in Figure 3.

An approach for designing a Haigh diagram for the multifunctional forceps-scissors (MFS) under cyclic load is proposed, by taking into account the influence of the stress ratio on the fatigue limit, as well as the nonlinearelastic crack growth threshold, as shown in Fig. 4. This method uses minimum of easily obtainable $\mathrm{Ti}-55$ material parameters of the MFS and permits an easy application of damage-tolerant design concepts when dimensioning for infinite fatigue life, as a central tool for stress based design computations. The numerical work was carried out using Anssy/Explicit code in order to develop the 


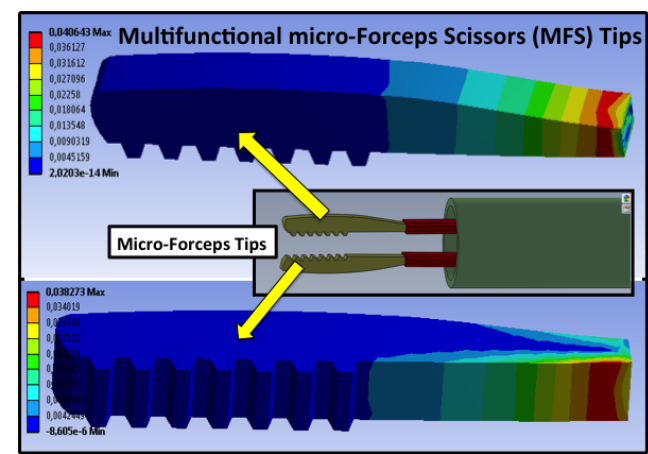

Fig. 3. Von Mises plasticity data of MFS.

\section{Haigh diagram.}

The mechanical behavior of material subjected to alternating loads can lead to failure even if the stresses in the material are much lower than the yield stress. Such a failure is known as fatigue failure and it is one of the most usual reasons for fracture [6]. A Haigh diagram is given in Fig. 4 to display the fatigue limit as a function of the mean stress. For a real structure, the Haigh diagram must be "reduced", due to the fact that the design was not used in laboratory conditions, where disturbing factors appear, like surface irregularities, volume factor due to process and volume factor due to geometry [7].

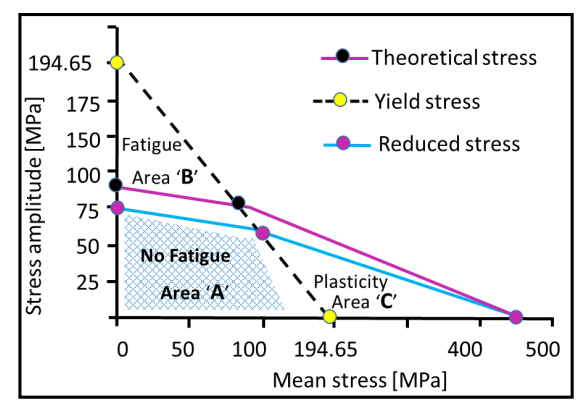

Fig. 4. Haigh diagram for fatigue life data of MFS specimen.

In Fig. 4., the Haigh diagram developed in this research, shows the safe and unsafe areas for the fatigue life prediction. Area 'A' shows the allowable service area, meaning that loading the forceps in such manner, that the stress does not cross the line, will ensure an almost infinite number of loading cycles to failure. Any values of stress above and close to the area 'A', would give a high number of loading cycles to failure (area ' $\mathrm{B}$ '). Above this area, fracture will occur after a while. The permanent plastic deformation will occur in area ' $\mathrm{C}$ '.

\section{Conclusions}

In this research, a method of experimental test and simulation study was proposed and examined to determine fatigue behavior. This approach is based on a combination of a real experimental testing, finite element analysis and fatigue prediction technique for $\mathrm{Ti}-55 \mathrm{ma}-$ terial of multifunctional forceps-scissor, used in medical engineering. The contribution of this work is concerned with theoretical modeling of MFS and provides a simple method for computing life cycle, using a minimum of easily obtainable material parameters for Haigh diagram. The numerical work was investigated using Ansys/Explicit code. The results show that there is a good agreement between experimental and numerical results. Although the fatigue parameters were estimated only approximately, the predictive capability of the presented methodology seems quite satisfactory and welcome.

\section{Acknowledgments}

This research was supported by Scientific Research Projects (BAP) of Istanbul University, Turkey, Project No: 24082

\section{References}

[1] D.F. Williams, Biomaterials 29, 2941 (2008).

[2] D. Kent, G. Wang, Z. Yu, X. Ma, M. Dargusch, J. of mech. beha. of biomed. mat. 4, 405 (2011).

[3] P.R. Singh, H. Lewis, Clinics Ger. Med. 22, 659 (2006).

[4] Y.L. Zhoua, M. Niinomi, T. Akahori, H. Fukui, H. Toda, Mat. Sci. and Eng. A 398, 28 (2005).

[5] M.N. Yuan, Y.Q. Yang, Z.J. Ma, X. Luo, B. Huang, Y. Chen, Scripta Materialia 56, 533 (2007).

[6] C.M. Sonsino, Intern. J. of Fatig. 29, 2246 (2007).

[7] T. Shimokawa, Y. Kakuta, Y. Hamaguchi, T. Aiyama, J. of Comp. Mat. 42, 655 (2008). 